\title{
23369
}

\section{European expert consensus on assessment and management of hospitalised exacerbations of COPD (CICERO ERS CRC)}

COPD - management, COPD - exacerbations, Nursing care

S. Ramakrishnan ${ }^{1}$, W. Janssens ${ }^{2}$, P. R. Burgel ${ }^{3}$, M. Contoli $^{4}$, F. M. E. Franssen ${ }^{5}$, N. Greening $^{6}$, T. Greulich ${ }^{7}$, I. Gyselinck ${ }^{2}$, A. Huerta ${ }^{8}$, J. Quint ${ }^{9}$, L. Van Fleteren ${ }^{10}$, H. Watz ${ }^{11}$, M. Bafadhel $^{1}$

${ }^{1}$ Respiratory Medicine Unit, Nuffield Department of Medicine, University of Oxford - Oxford (United Kingdom), ${ }^{2}$ Department of Respiratory Diseases, UZ Leuven, Research group BREATHE, KU Leuven - Leuven (Belgium), ${ }^{3}$ Faculty of medicine, Paris Descartes, CPSC - Paris (France), ${ }^{4}$ University of Ferrara, Department of Medical Sciences - Ferrara (Italy), ${ }^{5}$ Department of Respiratory Medicine, Maastricht University Medical Center - Maastricht (Netherlands), ${ }^{6}$ NIHR Leicester Biomedical Research Centre (Respiratory), Glenfield Hospital - Leicester (United Kingdom), ${ }^{7}$ Department of Medicine, Pulmonary and Critical Care Medicine, University Medical Centre Giessen and Marburg, Philipps-University, Member of the German Centre for Lung Research (DZL) - Marburg (Germany), ${ }^{8}$ IDIBAPS August Pi i Sunyer Biomedical Research Institute - Barcelona (Spain), ${ }^{9}$ National Heart \& Lung Institute, Imperial College - London (United Kingdom), ${ }^{10}$ Department of Internal Medicine and Clinical Nutrition at Institute of Medicine, SU Sahlgrenska - Göteborg (Sweden), ${ }^{11}$ Pulmonary Research Institute at LungenClinic Grosshansdorf, Airway Research Center North (ARCN), German Center for Lung Research (DZL) - Grosshandorf (Germany)

Introduction: Despite being a high-risk event for morbidity and mortality, there is little consensus globally regarding the assessment and management of hospitalised exacerbations of COPD.

Objectives: To find a consensus list of symptoms, physiological measures, clinical scores, patient questionnaires and investigations to be obtained at time of hospitalised COPD exacerbation and follow up.

Methods: A modified online Delphi survey of importance, feasibility and suggested frequency on Likert scales was undertaken. Consensus and stability criteria were pre-defined.

Results: 25 experts from 19 European countries participated. After completion of 2 rounds, consensus was achieved on 642 items (82\%). Experts achieved consensus quickly on history and physical examination items. There was less consensus on other items. For example (see table 1), many scores and 
questionnaires were not considered essential in clinical practice. Experts could not agree on pulmonary function tests to perform routinely at time of hospitalisation nor the importance or frequency in which to perform echocardiography, CT scan and pulmonary function tests at follow-up. Experts could not agree on duration of treatment

\begin{tabular}{|l|l|l|}
\hline Include & Consider & Exclude \\
\hline \hline & EWS & CODEX \\
& GCS & CIIS \\
& GOLD I-IV & ADO \\
MMRC & GOLD A-D & DOSE \\
CAT & BODEX & SGRQ \\
& HADS & SCOPEX \\
& CURB-65 & EXACT-Pro \\
& DECAF & BCSS \\
& VAS & Euro-QoL \\
\hline
\end{tabular}

Table 1: Consensus items for clinical scores and questionnaires at time of COPD exacerbation

Conclusion: Despite being a major health burden, hospitalised COPD exacerbations are assessed and managed variably across Europe. Standardisation could impact clinical outcomes.

Acknowledgements: This work was undertaken as part of the CICERO ERS clinical research collaborative. 